Voix et Images

voixetimages

\title{
Roman d'époque et fictions de notre époque
}

\section{Pascal Riendeau}

Volume 34, numéro 3 (102), printemps-été 2009

Trajectoires de l'auteur dans le théâtre contemporain

URI : https://id.erudit.org/iderudit/037670ar

DOI : https://doi.org/10.7202/037670ar

Aller au sommaire du numéro

\section{Éditeur(s)}

Université du Québec à Montréal

\section{ISSN}

0318-9201 (imprimé)

1705-933X (numérique)

Découvrir la revue

Citer ce compte rendu

Riendeau, P. (2009). Compte rendu de [Roman d'époque et fictions de notre époque]. Voix et Images, 34(3), 129-134. https://doi.org/10.7202/037670ar d'utilisation que vous pouvez consulter en ligne.

https://apropos.erudit.org/fr/usagers/politique-dutilisation/ 


\title{
R O M A N
}

\author{
Roman d'époque et fictions de notre époque \\ $++$
}

PASCAL RIENDEAU

Université de Toronto

Dans son essai Le rideau, Milan Kundera affirme que

\begin{abstract}
l'histoire d'un art (le "passé total» d'un art) est faite non seulement de ce que cet art a créé mais aussi de ce qu'il aurait pu créer, de toutes ses œuvres accomplies aussi bien que de ses œuvres possibles et non-réalisées [...]; il est resté de tous les «romans par lettres» un très grand livre qui a résisté au temps: Les liaisons dangereuses (1782) de Choderlos de Laclos $[\ldots]^{1}$.
\end{abstract}

Le jugement de Kundera paraît sévère mais assez juste dans l'ensemble. Ainsi est-il possible de se demander ce qu'un roman épistolaire peut encore nous proposer de nouveau au début du Xxie siècle. Peut-on réinventer un type de roman tombé en désuétude? Avec Cet océan qui nous sépare², Réal Ouellet n’a vraisemblablement pas cherché à donner une tournure inédite à son roman par lettres; il a plutôt choisi de reprendre les caractéristiques principales du genre, ce qui se justifie entre autres par l'époque durant laquelle se déroule le roman, la seconde moitié du XVII siècle. Ouellet tente de capter un certain esprit d'aventure et de découverte des mondes nouveaux qui pouvait animer alors les Européens. Un couple de jeunes mariés, Élisabeth et Christophe, quittent La Rochelle en septembre 1664 et s'embarquent pour la Martinique, car Élisabeth refuse d'épouser l'homme que lui avait destiné son père. Leurs principaux correspondants sont un couple d'amis, Béatrice et Maurice, restés en Bretagne. L'échange de lettres dure sept ans, durant lesquels surviennent la mort de la mère puis du père d'Élisabeth, la naissance de leurs trois enfants et le retour définitif de la famille en France. En revanche, après avoir tenté sa chance comme médecin en Martinique, Maurice décide d'y rester, mais sans Béatrice. En tout, plus d'une quinzaine de personnages participent à cette correspondance. Ces multiples épistoliers assurent une diversité de points de vue et un décentrement véritable: aucune voix ne domine. Ouellet n'a ajouté ni introduction, ni préface, ni

$$
+++
$$

1 Milan Kundera, Le rideau. Essai en sept parties, Paris, Gallimard, 2006, p. 194. 2 Réal Ouellet, Cet océan qui nous sépare, Québec, éditions 8, coll. «Contemporains», 2008, 254 p. 
note explicative; il n'a pas non plus créé de narrateur qui serait, comme chez Laclos, un «Rédacteur» pouvant expliquer comment cette correspondance a pu être réunie. Le rassemblement des lettres nous est donné tel quel, comme allant de soi. Cette absence de rédacteur évite de donner une seule interprétation des faits, du déroulement des événements ou des pensées qui parcourent les lettres de chacun.

Une des réussites du roman réside dans l'habileté de Ouellet à créer des voix distinctes, qu'on reconnaît aisément, sans qu'il ait besoin d'utiliser des artifices langagiers. On ne note pas de ruptures de ton, à peine quelques nuances dans l'énonciation qui nous permettent de bien distinguer chacun des personnages. Privilégiant une langue classique et un style sans fioritures, sans archaïsmes, l'auteur a surtout évité de tomber dans le piège de l'imitation de l'écriture épistolaire de cette époque. Seules les missives du jésuite François Fontaine, frère de Béatrice, paraissent plus stéréotypées. En filigrane, on remarque également que se dessine un portrait de l'émancipation féminine. Les deux jeunes femmes prennent leur destin en main et l'on sent clairement dans leurs lettres un désir de ne pas se voir confinées dans une position subalterne. Défier la loi paternelle, exiger l'égalité avec le nouveau mari sont des éléments qui caractérisent les discours et les actions d'Élisabeth et de Béatrice. À la fin du récit, chacune retourne à une occupation artistique qu'elle avait cru devoir abandonner: l'écriture pour la première et la peinture pour la seconde. Ouellet parvient à rendre crédible cette histoire de la quête d'un monde nouveau sans faire de ses personnages des héros épiques, sans exagérer la sensibilité de leurs discours. En réalité, les émotions sont plutôt contenues, même dans les moments les plus difficiles, comme le récit que fait Christophe de sa détention par les Espagnols, qui lui a presque été fatale.

Bien qu'il situe son roman au XviI siècle, Ouellet ne reprend pas les conventions du roman historique; quelques événements forment une toile de fond, mais celle-ci reste bien pâle. Il n'y a pas de trame historique. Ce qu'on retient surtout, c'est ce qui relève de l'histoire des mentalités, des idées et croyances de l'époque à propos de l'esclavage $-s^{\prime}$ il faut ou non y participer, s'il faut convertir les esclaves au christianisme - des horreurs de la guerre, du colonialisme et du désir des puissances coloniales de toujours vouloir posséder de nouveaux territoires. On ne sent jamais la voix de l'auteur qui viendrait s'imposer ou offrir une justification a posteriori; les personnages sont aux prises avec les opinions et les contradictions qui leur sont propres. Le roman ne privilégie pas non plus ce parti pris plus anthropologique où la rencontre avec l'Autre ou encore la relation avec les indigènes serait déterminante. Élisabeth et Christophe arrivent sur une terre qui est déjà française, ses premiers habitants, appelés «Sauvages caraïbes» dans le roman, ayant presque tous été chassés. Par contre, le territoire martiniquais devient une source constante de découvertes : la faune, la flore, mais aussi la nouvelle nourriture (banane, chocolat). D'ailleurs, une dizaine d'illustrations - assez naïves mais non sans charme - accompagnent les missives (parfois didactiques) sur les merveilles que l'on trouve à la Martinique, comme en témoigne une lettre d'Élisabeth écrite moins d'un an après son arrivée: "Celui que je préfère - et je ne sais pas s'il faut l'appeler un fruit - est une espèce de gros artichaut qui ressemble à une pomme de pin géante [...]. Certains l'appellent le roi des fruits parce qu'il porte une couronne. Son vrai nom est l'ananas.» (26) 
Si la forme est classique et maîtrisée chez Ouellet, dans Matamore $n^{\circ} 29^{3}$, le premier roman d'Alain Farah, elle est éclatée mais plus hésitante. Dans cette œuvre de fiction qu'on pourrait qualifier d'expérimentale - si le terme n'était pas galvaudé -, l'auteur laisse place à quelques expérimentations formelles et joue avec différents codes assez communs aujourd'hui : fragmentation, dissertation, aphorisme, autofiction. Le roman est divisé en vingt-neuf chapitres - ce n'est pas une surprise: un rappel du titre, de l'âge du narrateur à la fin de son récit - et, dans une large part, composé de paragraphes très courts, souvent d'une seule phrase, d'une seule petite ligne, ce qui crée à l'occasion des effets de poésie en prose. Le rythme est rapide, saccadé; on change brusquement de lieu, d'univers. En retour, il faut dire que la narrativité reste minimale. Le développement du récit principal se fait de façon aléatoire, suit sa propre logique étonnante et il est constamment interrompu par des considérations diverses du narrateur ou encore par un autre récit qui apparaît soudainement et nous entraîne ailleurs. Que raconte Matamore $n^{\circ} 29$ ? C'est l'histoire d'«un livre, dit le narrateur, que je suis sur le point de terminer, un livre où je consigne les expériences "spéciales" vécues entre mes vingt-cinq et mes vingt-neuf ans» (150). En fait, plus que le récit des expériences, c'est davantage le récit du livre que nous suivons, de toutes les façons possibles de consigner les expériences afin d'en arriver à un livre. Le narrateur s'observe quand il écrit, commente son écriture, s'en amuse et partage avec le lecteur ses interrogations, ses constatations, comme lorsqu'il affirme au dixième chapitre, de façon candide ou ironique, «qu'il ne sait toujours pas de quoi parle son texte» (64).

Sous-titré «Mœurs de province», à l'instar de Madame Bovary, le récit de Farah n'a apparemment pas repris d'autres éléments de la poétique romanesque de Flaubert. En fait, ce petit clin d'œil relève plutôt d'un des multiples déplacements qui traversent l'ensemble de Matamore $n^{\circ} 29$, qu'ils soient géographiques, culturels ou identitaires. Le narrateur (il s'appelle Alain ou M. Farah, mais jamais Alain Farah) et son alter ego (ou son alibi), Joseph Mariage, convoqué dès l'incipit, sont des personnages changeants: primesautier ou sermonneur pour le premier, énigmatique et insaisissable pour le second. On comprend toutefois que ce narrateur est un étudiant qui connaît bien la littérature, s'intéresse à l'art et fait preuve d'un certain militantisme politique anti-Bush (le président américain est surnommé le "Potentat Buisson»). Cet étudiant québécois, dont les ancêtres sont égyptiens, après avoir parcouru sa "province», se retrouve plus tard à Paris où il rencontre notamment un écrivain français fort connu prénommé Alain, à l'origine du nouveau roman. Tout cela ne tient évidemment pas compte des ruptures temporelles, des invraisemblances, des anachronismes, du refus de toute organisation chronologique fiable. Le récit affiche pourtant une unité par sa tonalité, son style. Voici un exemple, presque choisi au hasard, qui montre à la fois le rythme du récit, le questionnement identitaire souvent ironique, l'humour, les situations absurdes, ainsi que les idées de déplacement et d'incertitude :

$$
++
$$

3 Alain Farah, Matamore no 29. Mours de province, Montréal, Le Quartanier, coll. «Série QR», 2008, 217 p. 
Je suis devant le musée.

C'est étrange, j'ai oublié d'où je viens.

Mes pieds sentent les fleurs, en tout cas.

Où vis-je?

Sûrement pas à Los Angeles.

Où vais-je?

Au musée oui ce musée où un jour je m'étais recueilli sur la tombe d'une volaille.

Ma tête va éclater. (82)

L'auteur multiplie les digressions, les coq-à-l'âne; d'autres associations de phrases donnent l'impression d'avoir été tirées d'un cadavre exquis. Tout semble propice à faire dérailler le récit ou à l'entraîner ailleurs. Parmi les digressions les plus caractéristiques, on remarque quelques petites leçons, qui portent sur l'origine du mot tennis et la façon dont on doit y compter les points, sur le patapore ou canon à patates - tiens, une allusion à Boris Vian - ou sur les poissons (la sole, le lieu) que nous connaissons mal, paraît-il. Une assez longue étude sur Joyce présentée comme une envolée se transforme en une performance du narrateur devant un écrivain français (un autre) étonné et moqueur. L'autodérision dont le narrateur fait alors preuve n'est qu'une des formes d'humour du texte où se multiplient les commentaires ironiques, les calembours ou autres jeux de mots. Les créations langagières et les jeux textuels dans le premier roman d'Alain Farah sont parfois amusants ou intelligents, parfois faciles ou facétieux, et ils montrent assez rapidement la limite de ces expérimentations formelles et les risques inhérents à tenter de créer une forme de fiction inédite.

Dans La bibliothèque de Sodome ${ }^{4}$, recueil de nouvelles de Louis-Philippe Hébert, on se trouve toujours légèrement dépaysé, c'est-à-dire que ce qui semble familier ne l'est jamais tout à fait. Chaque nouvelle présente un personnage singulier, un témoin essentiel, ou encore elle fait le récit d'une histoire morbide, d'une obsession (une simple casquette ou une hypothèse scientifique), d'un moment de folie inexplicable. Les univers dépeints oscillent entre le noir et le lumineux, le lugubre et l'insouciant; la mort est toujours présente, le meurtre aussi. L'auteur recourt aux procédés du fantastique pour rendre crédibles des situations banales ou quotidiennes qui se transforment soudainement, mais il emprunte également à l'imaginaire littéraire traditionnel québécois, celui des contes et des légendes qui n'auraient pu se passer du Diable ou d'un de ses avatars, sans pourtant que le bon Dieu ou de valeureux curés ne viennent prendre la défense des pauvres âmes en danger. Hébert ne s'aventure pas sur ce terrain du merveilleux qui consisterait à faire du Diable un personnage, mais sa présence paraît indéniable - «Ne dit-on pas que le Diable est dans les détails?» (115) - aux yeux de ceux dont les actions peuvent sembler incom-

$$
+++
$$

4 Louis-Philippe Hébert, La bibliothèque de Sodome, Montréal, Les Herbes rouges, coll. «Nouvelles », 255 p. 
préhensibles ou injustifiables. Hébert ne tente pas de nous entraîner vers une "connaissance du mal»; ses nouvelles ne sont pas des allégories du mal. Victimes et bourreaux se confondent parfois, y compris dans les chutes ironiques qui empêchent un simple retour à l'ordre établi ou alors quand celui-ci advient, il paraît dérisoire. Il n'y a pas non plus de rédemption ou de justice. Mais sommes-nous réellement, comme le prétend l'éditeur, «aux limites de l'obscène»? La sexualité occupe-t-elle une place déterminante dans La bibliothèque de Sodome? À vrai dire, la sexualité hante toujours l'atmosphère des nouvelles, mais dans quelques-unes, elle reste plus discrète, refoulée ou sous-entendue. Quant à l'obscénité, elle n'est pas la seule convoquée et elle partage l'espace avec l'abjection, la sordidité ou la monstruosité, qui visent toutes à étonner, voire à secouer le lecteur. Les déplacements vers l'abject ou le morbide se produisent souvent de façon inattendue, mais même dans ces circonstances, Hébert maintient un écart entre la réalité décrite et le langage de cette description, qui reste sobre et soigné, à quelques exceptions près.

Tout en étant bien ancré dans son époque, La bibliothèque de Sodome propose aussi une critique du monde actuel, en particulier dans la nouvelle éponyme. Le directeur de la nouvelle bibliothèque nationale est un homme, nous dit l'incipit, " qui en voulait à son époque", qu'il dénonce avec un certain cynisme, du moins avec des propos assez convenus sur la société, les politiciens, les lecteurs. Ce qui rend ce personnage plus original, c'est l'obsession qu'il développe pour un lecteur, son lecteur, celui qui a emprunté son nouveau roman érotique, Ardente caresse, placé sur la table au milieu de Sade, Bataille, Arsan et Réage. Son obsession - on s'en doute - lui sera fatale. Quant à «Une idée renversante», elle n'exploite pas tant l'obscénité que la scatologie. En tant que témoin de la logique étonnante d'un scientifique à la dérive, un médecin raconte les derniers moments de son patient, Marc Mariner, professeur de physique qui semble avoir reçu une drôle de pomme newtonienne sur la tête. Un jour, il a une illumination théorique qu'il s'empresse de mettre en pratique: la réversibilité nutritionnelle. Convaincu qu'il lui revient d'en faire l'expérience, il cessera de se nourrir de façon conventionnelle, remettra dans son corps, par le même orifice, ses propres selles et boira son urine - aucun autre moyen efficace de réversibilité ne lui semblait alors possible. Il deviendra scatophage, se nourrissant d'excréments de chiens, de chats et de fientes d'oiseaux. Grâce au témoignage du médecin, on apprend que la maladie originale dont il souffrait porte maintenant son nom. Recourir à la scatologie, est-ce encore un moyen de susciter l'aversion? L'ironie d'Hébert le suggère, car le narrateur, avant d'arriver au cœur du propos, croit se douter que des lecteurs ne le suivront pas et avertit les plus sensibles de s'abstenir de lire la suite... Il est possible que la nouvelle dégoûte quiconque tolère mal le discours excrémentiel, mais elle est narrée de manière rigoureuse et précise, sans complaisance évidente, en limitant les détails.

Seule la dernière nouvelle, "Le ver est dans la pomme», s'engage du côté de la description sexuelle explicite, celle d'une femme dans un moment d'attente, puis d'extase. Ce texte propose ironiquement un retour à Sodome puisque la protagoniste, réveillée durant la nuit par le retour improbable de son mystérieux amant, se laisse conduire, puis s'installe sur le plancher de la cuisine, avant d'être attachée et sodomisée par l'homme: "Quand ses fesses s'écartèrent exagérément, et que cette 
exagération alla jusqu'à la brûler, elle conclut qu'il cherchait à l'empaler plus qu'à la baiser.» (241) Si nous ne connaissons que le point de vue du personnage féminin (ses fantasmes, ses désirs, ses sensations), la façon de le décrire, on le constate, reste éminemment masculine. Hébert ne semble pas tant explorer un côté plus caché de la sexualité féminine que de tisser des liens entre ce qu'il appelle «la transgression», le désir et la violence ou encore le plaisir, l'inceste et la mort. Tout cela, d'ailleurs, n'est pas sans rappeler Bataille: «Que signifie l'érotisme des corps sinon une violation de l'être des partenaires ${ }^{5}$ ?" Si la protagoniste occupe presque tout l'espace de la nouvelle - l'homme n'est qu'un figurant - , elle prend peu la parole. Même lorsqu'elle raconte l'histoire qui aurait tout fait basculer quand elle avait 12 ans, c'est le narrateur qui prend en charge la quasi-totalité du récit. Encore une fois, Hébert convoque le fantastique - ou du moins l'inexpliqué - , car il devient presque impossible de départager ce qui relève du rêve, du délire alcoolique, du fantasme et de la réalité. Le passé et le présent se confondent et elle se demande comment l'homme qu'elle a aimé, mais qu'elle croyait mort, peut être de retour. Pourquoi est-il muet? Comment expliquer la présence de l'absent, du disparu?

Tout compte fait, seule cette chronique rassemble ces trois ouvrages qui autrement s'opposent, tant par la forme, le style que l'imaginaire. Le roman de Réal Ouellet est anachronique, alors que les deux autres, à leur façon, sont des manifestations singulières de leur époque. Cet océan qui nous sépare ne renouvelle pas la forme désuète du roman épistolaire, mais celui-ci, grâce à l'écriture précise et soignée de Ouellet, conserve une certaine pertinence pour penser la vie des siècles passés sans recourir au roman "historique». Matamore nº 29 est appelé roman par l'éditeur, sans doute parce qu'aucun autre terme ne pouvait décrire cet assemblage textuel de récits, de fragments et de pensées. Alain Farah cherche une forme littéraire originale qu'il ne semble pas avoir véritablement trouvée; c'est une œuvre inachevée, inaboutie. En respectant les paramètres de la nouvelle et du fantastique et en reprenant des thèmes analogues d'une nouvelle à l'autre, Louis-Philippe Hébert donne à son recueil une grande cohérence. Sans trop se donner le rôle de l'auteur provocateur, Hébert, par son désir de repousser des interdits et d'exploiter certaines transgressions dans La bibliothèque de Sodome, critique implicitement son époque en lui demandant de faire face à ce qu'elle peut encore juger dérangeant ou répulsif, y compris dans la fiction. 